\title{
Solution of Poisson Equation using Isogeometric Formulation
}

\author{
Sang Jin LEE
}

ADOPT Research Group, Department of Architectural Engineering, Gyeongsang National University

\begin{abstract}
Isogeometric solution of Poisson equation is provided. NURBS (NonUniform B-spline Surface) is introduced to express both geometry of structure and unknown field of governing equation. The terms of stiffness matrix and load vector are consistently derived with very accurate geometric definition. The validity of the isogeometric formulation is demonstrated by using two numerical examples such as square plate and L-shape plate. From numerical results, the present solutions have a good agreement with analytical and finite element (FE) solutions with the use of a few cells in isogeometric analysis.
\end{abstract}

Keywords : Isogeometric Analysis, Poisson Equation, Partial Differential Equation, NURBS, Knot Vector, Control Point, Plate

\section{INTRODUCTION}

Nowadays, isogeometric analysis (IGA) is being recognized as to-be essential analysis technique in various engineering fields and it is now rapidly introduced in science and engineering problems. To begin with the use of isogeometric concept, both clear understanding of NURBS and the way how to treat structural geometry modeled by using NURBS are necessarily required. Isogeometric formulation uses tricky B-spline which is somehow quite difficult to understand outside of computer screen where interactive manipulation is normally not possible.

On the contrary, it is very fortunate that isogeometric formulation can be categorized as a branch of FE method since it is very similar to the FE isoparametric formulation (Irons and Zienkiewicz, 1968).

Corresponding Author: Sang Jin LEE, Professor

Department of Architectural Engineering, Gyeongsang

National University

Gajwadong, Jinjusi, Gyeongsangnamdo, 660-701, Korea

Tel :+82 557516541 e-mail: lee@gnu.ac.kr

Construction Technology Research \& Development Program

(PN: 06-R\&D-B03) are gratefully acknowledged and sincere appreciation to SJ-MIRAE for their providing CAGD technology for this research.

This is an Open Access article distributed under the terms of the Creative Commons Attribution NonCommercial License (http://creativecommons. org/licenses/bync/3.0/) which permits unrestricted noncommercial use, distribution, and reproduction in any medium, provided the original work is properly cited.
The use of spline in FE formulation can be found in literatures around 1980's (Mizusatia et al., 1979; Zamani, 1981) and we can also find a basic application of B-spline to structural analysis in architectural engineering literature (Kim, 1984). However, the present form of isogeometric formulation appears to be very popular due to the Hughes's work (Hughes et al., 2005). With series of his research works on isogeometric formulation, he recently published meaningful results on the IGA with his colleague (Cottrell et al., 2009).

In solving engineering problem, this concept is still new and is just being introduced. So, there are many aspects of IGA which is required to be explored. In particular, the versatility and generality of this method can be only guaranteed by its applications to various engineering problems and subsequent detailed parametric analyses on certain physical problem.

In this context, the Poisson equation with Dirichlet boundary condition is here solved by isogeometric concept. As well-known fact, the Poisson equation $-\nabla^{2} \mathrm{u}=\mathrm{f}$ is simplest elliptic partial differential equation (PDE). For two-dimensional problem, the load function is applied to the domain denoted by $\Omega \in \mathrm{R}^{2}$ and a solution of Poisson equation has to satisfy the essential boundary conditions on the boundary $\partial \Omega$. In this study, $\mathrm{u}$ is considered as temperature in the domain $\Omega$ subjected to the external heat load $\mathrm{f}$.

\section{B-SPLINES}

\subsection{Knot vector}

Knot vector is a set of coordinates in the parametric space and it is written as $\Xi=\left\{\xi_{1}, \xi_{2}, \cdots, \xi_{n+p+1}\right\}$, where $\xi_{i} \in \mathrm{R}^{1}$ is the $\mathrm{i}^{\text {th }}$ knot, $\mathrm{n}$ is 
number of basis functions and $\mathrm{p}$ is the order of basis function.

\subsection{Basis function}

B-spline basis functions are defined recursively (de Boor, 1987) and it begin with order $\mathrm{p}=0$ such as

$$
\mathrm{N}_{\mathrm{i}, 0}(\xi)= \begin{cases}1 & \text { if } \xi_{\mathrm{i}} \leq \xi<\xi_{\mathrm{i}+1} \\ 0 & \text { otherwise }\end{cases}
$$

and for higher order $\mathrm{p}=1,2,3, \cdots$, it is defined as

$$
\mathrm{N}_{\mathrm{i}, \mathrm{p}}(\xi)=\frac{\xi-\xi_{\mathrm{i}}}{\xi_{\mathrm{i}+\mathrm{p}}-\xi_{\mathrm{i}}} \mathrm{N}_{\mathrm{i}, \mathrm{p}-1}(\xi)+\frac{\xi_{\mathrm{i}+\mathrm{p}+1}-\xi}{\xi_{\mathrm{i}+\mathrm{p}+1}-\xi_{\mathrm{i}+1}} \mathrm{~N}_{\mathrm{i}+1, \mathrm{p}-1}(\xi)
$$

The B-spline function with uniform knot vector ${ }^{\dagger}$ for order $\mathrm{p}=0,1$, 2 can be illustrated as shown in Figure 1.

Note that the functions with non-uniform knot vector are also important to create curvy or complex structural geometry in engineering problem.

\subsection{Surface definition}

For two dimensional structural problems, the B-spline surface can be defined by a tensor product such as

$$
\begin{aligned}
S(\xi, \eta) & =\sum_{i=1}^{n} \sum_{j=1}^{m} N_{i, p}(\xi) M_{j, q}(\eta) C_{i, j} \\
& =\sum_{i=1}^{n} \sum_{j=1}^{m} \widetilde{N}_{i, p ; j, q}(\xi, \eta) C_{i, j}
\end{aligned}
$$
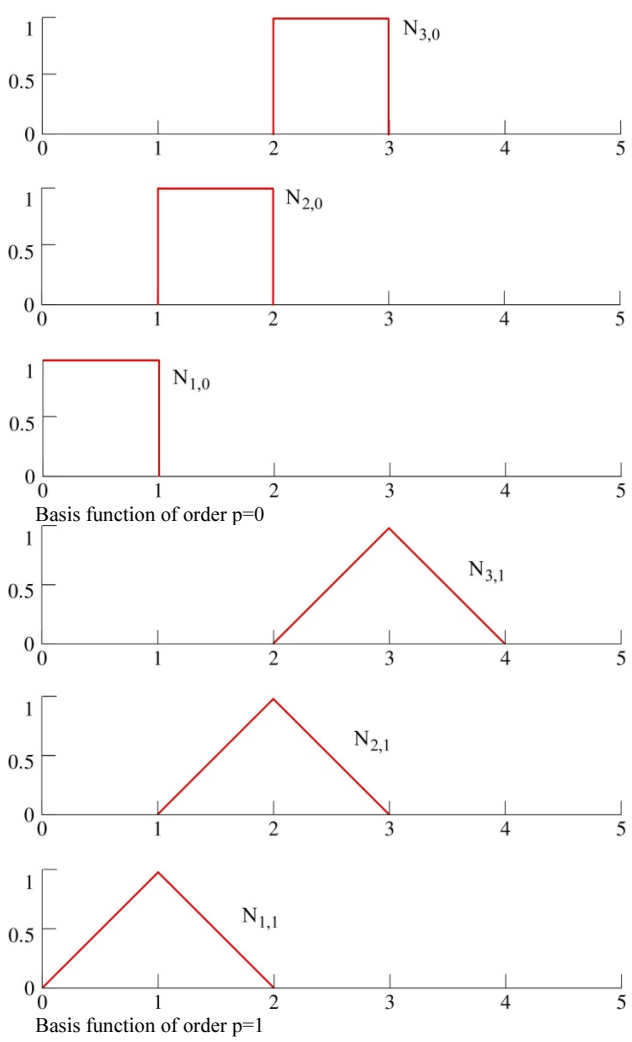

${ }^{\dagger}$ Uniform knot vector: the knots are equally spaced in the parametric space.
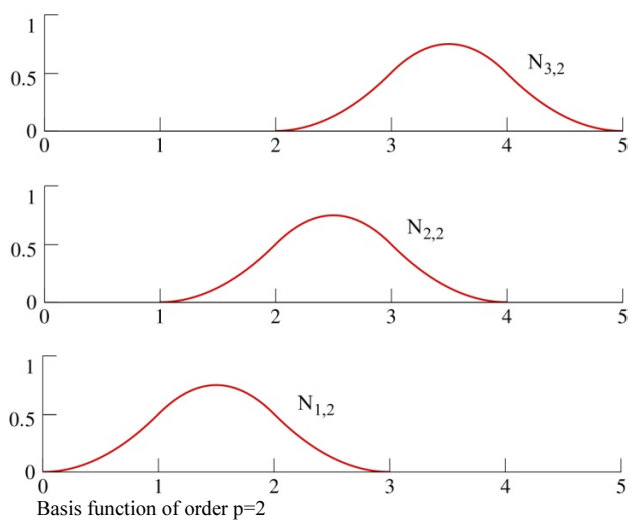

Figure 1. Basis function of order $\mathrm{p}=0,1$ and 2 for uniform knot vector $\Xi=\left\{\xi_{1}, \xi_{2}, \xi_{3}, \xi_{4}, \xi_{5}, \ldots\right\}=\{0,1,2,3,4, \ldots\}$

where the $n \times m$ control point net with control points $C_{i, j}$ and polynomial order p,q and knot vectors $\Xi=\left\{\xi_{1}, \xi_{2}, \cdots, \xi_{n+p+1}\right\}$ and $\Theta=\left\{\eta_{1}, \eta_{2}, \cdots, \eta_{\mathrm{m}+q+1}\right\}$ are used. $\mathrm{N}_{\mathrm{i}, \mathrm{p}}(\xi)$ and $\mathrm{M}_{\mathrm{i}, \mathrm{q}}(\eta)$ are univariate $\mathrm{B}$-spline basis functions of order $\mathrm{p}$ and $\mathrm{q}$, corresponding to knot vectors $\Xi$ and $\Theta$ respectively.

\section{ISOGEOMETRIC FORMULATION}

\subsection{Strong form}

The strong form of Poisson equation as boundary value problem (BVP) can be defined as

$$
-\nabla^{2} \mathrm{u}(\mathrm{x})=\mathrm{f}(\mathrm{x}) \text { for all } \mathrm{x} \in \Omega \text {. }
$$

A solution of above equation has to satisfy boundary condition on the boundary $\partial \Omega$

$$
\alpha \mathrm{u}+\beta \frac{\partial \mathrm{u}}{\partial \mathrm{n}}=\mathrm{g} \text { on } \partial \Omega
$$

where $\partial \mathrm{u} / \partial \mathrm{n}$ denotes the derivatives in the direction normal to the boundary $\partial \Omega$ and $\alpha$ and $\beta$ are constant although the variable coefficients are also possible. If the constant $\beta=0$, then the BVP is referred to Dirichlet problem and if the constant $\alpha=0$, we have Neumann problem. In this study, we will consider only Dirichlet problem using isogeometric concept.

\subsection{Weak form}

The weak form of Poisson equation can be derived by multiplying both side by a test function, integrating over the domain $\Omega$, and performing integration by parts. The result is

$$
\int_{\Omega} \nabla \mathrm{w} \nabla \mathrm{ud} \Omega=\int_{\Omega} \mathrm{wf} \mathrm{d} \Omega
$$

for all test function w.

we can rewrite the above equation in concise form so that the weak form of the above equation becomes

$$
\mathrm{a}(\mathrm{w}, \mathrm{u})=\mathrm{L}(\mathrm{w})
$$

where 


$$
\begin{aligned}
\mathrm{a}(\mathrm{w}, \mathrm{u}) & =\int_{\Omega} \nabla \mathrm{w} \nabla \mathrm{ud} \Omega \\
\mathrm{L}(\mathrm{w}) & =\int_{\Omega} \mathrm{wf} \mathrm{d} \Omega .
\end{aligned}
$$

\subsection{Galerkin method}

Galerkin method is used to turn the weak statement of the problem into a system of algebraic equations. The relevant derivations take place in finite-dimensional subspace. In this study, the subspaces are defined using the isoparametric NURBS basis:

$$
w^{h}=\sum_{a=1}^{n_{e q}} N_{a} d_{a} .
$$

Furthermore, the function $\mathrm{g}^{\mathrm{h}}$ is given similarly by quantities $\mathrm{g}_{\mathrm{a}, \mathrm{a}}=1, \ldots, \mathrm{n}_{\mathrm{nb}}$

$$
g^{h}=\sum_{a=n_{e q}+1}^{n_{n b}} N_{a} g_{a} .
$$

Therefore, trial functions can be expressed as

$$
u^{h}=\sum_{a=1}^{n_{e q}} N_{a} d_{a}+\sum_{a=n_{e q}+1}^{n_{n b}} N_{a} g_{a} .
$$

where $n_{e q}$ is number of equation and $n_{n b}$ is number of boundary condition.

Substituting (8), (10) into the weak form yields

$$
\sum_{\mathrm{a}=1}^{\mathrm{n}_{\mathrm{eq}}} \mathrm{d}_{\mathrm{a}}\left(\sum_{\mathrm{b}=1}^{\mathrm{n}_{\mathrm{eq}}} \mathrm{a}\left(\mathrm{N}_{\mathrm{a}}, \mathrm{N}_{\mathrm{b}}\right) \mathrm{d}_{\mathrm{b}}=\mathrm{L}\left(\mathrm{N}_{\mathrm{a}}\right)-\mathrm{a}\left(\mathrm{N}_{\mathrm{a}}, \mathrm{g}^{\mathrm{h}}\right)\right)=0
$$

As the $\mathrm{d}_{\mathrm{a}}$ are arbitrary and the following equation can be derived as

$$
\sum_{\mathrm{b}=1}^{\mathrm{n}_{\mathrm{eq}}} \mathrm{a}\left(\mathrm{N}_{\mathrm{a}}, \mathrm{N}_{\mathrm{b}}\right) \mathrm{d}_{\mathrm{b}}=\mathrm{L}\left(\mathrm{N}_{\mathrm{a}}\right)-\mathrm{a}\left(\mathrm{N}_{\mathrm{a}}, \mathrm{g}^{\mathrm{h}}\right)
$$

where the terms $\mathrm{K}_{\mathrm{ab}}$ and $\mathrm{F}_{\mathrm{a}}$ can be defined as

$$
\begin{gathered}
\mathrm{K}_{\mathrm{ab}}=\mathrm{a}\left(\mathrm{N}_{\mathrm{a}}, \mathrm{N}_{\mathrm{b}}\right) \\
\mathrm{F}_{\mathrm{a}}=\mathrm{L}\left(\mathrm{N}_{\mathrm{a}}\right)-\mathrm{a}\left(\mathrm{N}_{\mathrm{a}}, \mathrm{g}^{\mathrm{h}}\right)
\end{gathered}
$$

and in matrix form,

$$
\begin{gathered}
\mathbf{K}=\left[\mathrm{K}_{\mathrm{ab}}\right] \\
\mathbf{F}=\left\{\mathrm{F}_{\mathrm{a}}\right\} \\
\mathbf{d}=\left\{\mathrm{d}_{\mathrm{a}}\right\} .
\end{gathered}
$$

For $\mathrm{a}, \mathrm{b}=1, \ldots, \mathrm{n}_{\text {eq }}$, we can finally have algebraic equation such as

$$
\mathbf{K d}=\mathbf{F}
$$

where $\mathrm{K}$ is the stiffness matrix and $\mathrm{F}$ is load vector.

\subsection{Some remarks on isogeometric implementation.}

The isogeometric formulation is very similar to the FE formulation. The only difference between IGA and FE analysis is the functions they are using in the formulation in order to express the geometry and solution field of the problem. IGA uses the B-spline basis functions in the formulation while polynomial function such as Lagrange polynomial is used in FE formulation. As described in Section 2.3, all the quantities are represented through control points in isogeometric formulation. It means that the stiffness term will be formed according to control point net information but numerical integration will take place with the mesh created by knot vector.

For visual explanation on isogeometric formulation, we illustrate a schematic diagram of isogeometric concept in Figure 2. In this diagram, the isogeometric mesh is formed by Cell I and Cell II (or Element I and II). To deal with this new concept, first, we have to know that IGA have transformation between physical domain and parametric domain. The physical domain is represented by the control point net as shown in Figure 2 and isogeometric mesh can be represented in both physical domain and parametric domain.
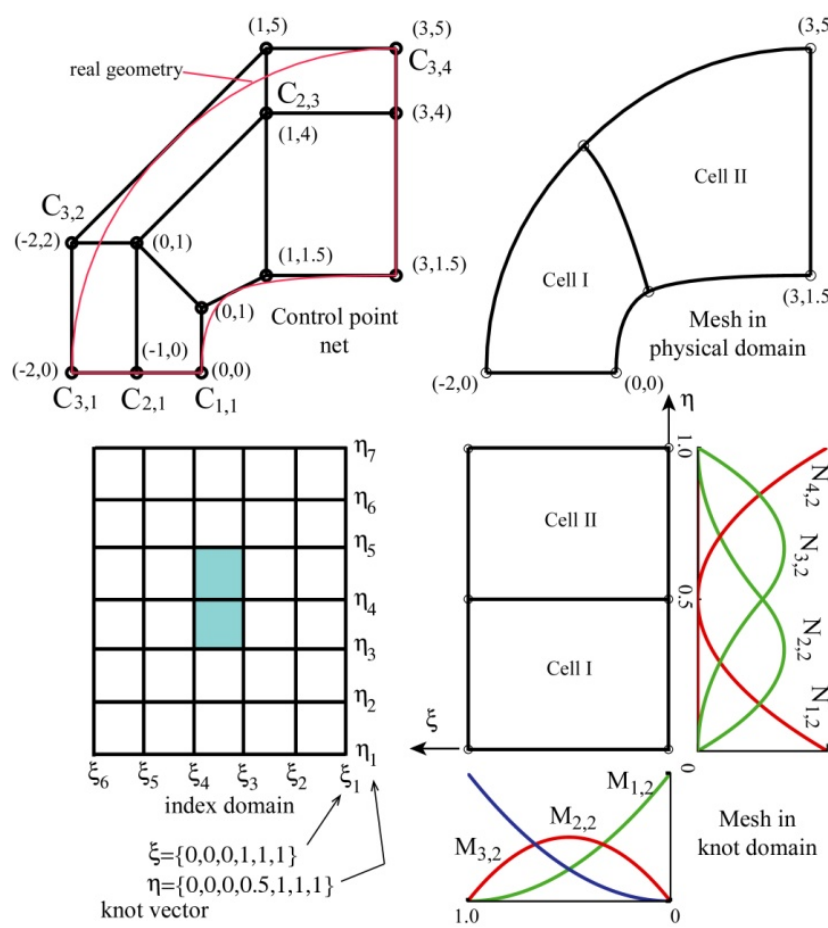

Figure 2. Schematic diagram for isogeometric analysis: the way how to express the geometry of the structure using control point net and the relationship between the physical domain and knot domain.

One of the most confusing aspects in isogeometric formulation could be the possibility that control point net and cell (or element) does not exactly match in physical domain. Therefore, for computer implementation, we should detect which control point is related to the cell we are going to integrate. Apart from this detection process, it is quite straightforward to form the stiffness matrix numerically since IGA uses the same numerical integration procedure normally used in FE method. For example, when we create the stiffness term for Cell I, we have to find out which basis function is related to Cell I. From Figure 2, we can see that the basis functions $\mathrm{N}_{1,2}, \mathrm{~N}_{2,2}, \mathrm{~N}_{3,2}$ and 
$\mathrm{M}_{1,2}, \mathrm{M}_{2,2}, \mathrm{M}_{3,2}$ are related to Cell $\mathrm{I}$. In other words, control points $\mathrm{C}_{1}$ ${ }_{11}, \mathrm{C}_{2,1}, \mathrm{C}_{3,1}, \mathrm{C}_{1,2}, \mathrm{C}_{2,2}, \mathrm{C}_{3,2}, \mathrm{C}_{1,3}, \mathrm{C}_{2,3}, \mathrm{C}_{3,3}$ are directly associated with Cell I. The Poisson equation has only one solution field for each control point so that it is considered as the problem with single degree of freedom per control point. Therefore, the $9 \times 9$ stiffness matrix $K_{9 \times 9}^{1}$ is formed for Cell I and subsequently $\mathrm{K}_{9 \times 9}^{\mathrm{II}}$ will be calculated for Cell II. The element stiffness $\mathrm{K}^{\mathrm{I}}$ and $\mathrm{K}^{\mathrm{II}}$ is then assembled by the same assembly rule used in FE method (Hughes, 1987). In this problem, we finally have $12 \times 12$ stiffness matrix $K_{12 \times 12}^{\text {structure }}$ for the structure which is modeled by 12 control points. Note that control point of IGA is the counterpart of nodal point in FE method. Hence, the unknown field $\mathrm{d}_{\mathrm{a}}$ of $(13 \mathrm{e})$ is representing the quantity at control points. Therefore, we need to find out true solutions in the domain $\Omega$ using (3).

\section{NUMERICAL TEST}

\subsection{Square plate}

Square plate with uniform load function $\mathrm{f}(\mathrm{x})=1$ with Dirichlet boundary condition is considered. The geometry of the plate is illustrated in Figure 3.

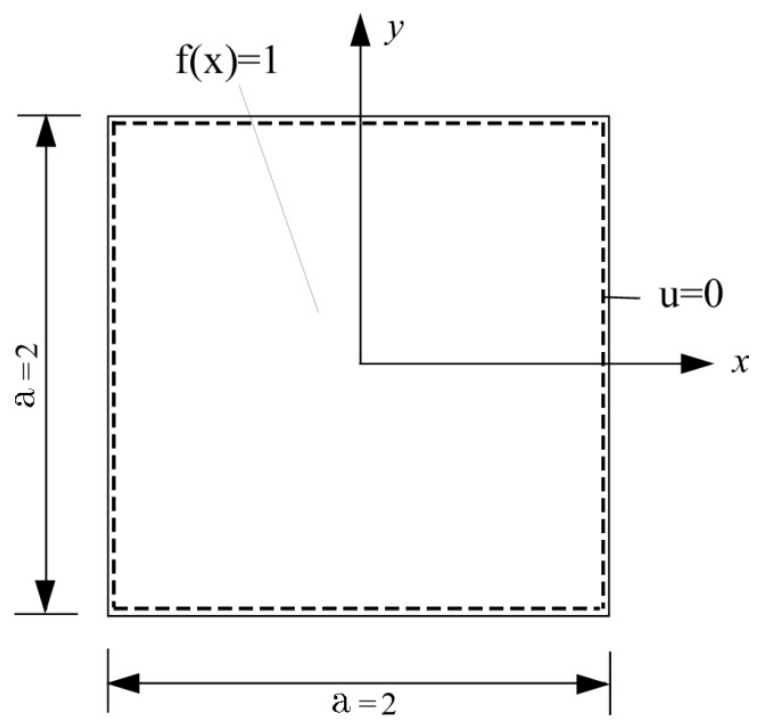

Figure 3. Square plate subjected to uniform heat load with Dirichlet boundary condition

This problem represents a simple diffusion model for the temperature distribution $\mathrm{u}(\mathrm{x}, \mathrm{y})$ in a square plate. The load is here considered as uniform heating of the square plate. The boundary condition models the edge of the plate being kept at zero temperature so that $\mathrm{u}(-1, \mathrm{y})=\mathrm{u}(1, \mathrm{y})=\mathrm{u}(\mathrm{x},-1)=\mathrm{u}(\mathrm{x}, 1)=0$.

Prior to IGA, we prepare reference solutions for comparison. Two reference solutions such as analytical and FE solutions are prepared. The analytical solution we adopted here in comparison is a series solution as follows

$$
\begin{aligned}
& u(x, y)=\frac{\left(1-x^{2}\right)}{2}-\frac{16}{\pi^{3}} \sum_{\substack{k=1 \\
\text { k odd }}}^{\infty}\left\{\frac{\sin (k \pi(1+x) / 2)}{k^{3} \sinh (k \pi)}\right. \\
& x(\sinh (k \pi(1+y) / 2)+\sinh (k \pi(1-y) / 2))\} .
\end{aligned}
$$

We produce the temperature distribution using the above equation and illustrated in Figure 4. The maximum temperature at centre of plate is turned out to be 0.29469 .

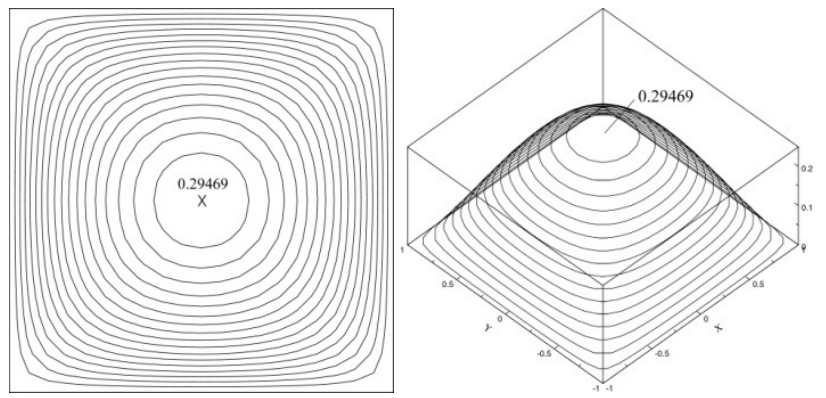

Figure 4. Analytical solution of square plate

The FE solutions are also produced by using bilinear and biquadratic standard FEs (Q1 and Q2). For Q1 FE, 16×16 FE mesh is used in the analysis and $8 \times 8 \mathrm{FE}$ mesh is used for Q2 FE as shown in Figure 5 . Both FEs produces very similar solutions: Q1 and Q2 produce the values of 0.2956 and 0.29468 respectively at the centre of plate.

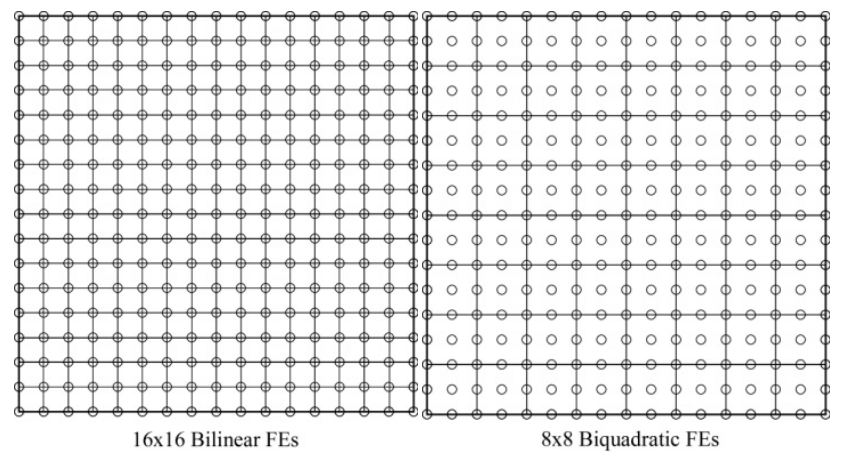

Figure 5. FE meshes of square plate with (left) bilinear FE (Q1) and (right) biquadratic FE (Q2)

For the initial IGA, we use $2 \times 2$ control points net with only one cell as illustrated in Figure 6 (Case I). The degree of basis function is chosen as $\mathrm{p}=2$ and uniform knot vector $\Xi=\Theta=\{0,0,0,1,1,1\}$ is used in both $\xi$ and $\eta$ directions which mean that the spline in both directions are given as open knot splinet. Therefore, the square plate is parameterized with the continuity of $\mathrm{C}^{1}$. It should be noted that the NURBS surface is modeled with equal weight in this study. For further analysis, we modify the control point net and knot vector as shown in Figure 6. We just increase the number of control points and insert the knot to the geometry definition. Only a simple modification is therefore required for h-adaptivity in IGA. The control points and knot vector used in this example is summarized in Table 1.

Table 1. Control points and knot vector

\begin{tabular}{c|l|l}
\hline Case & Control points (C) & \multicolumn{1}{|c}{ Knot vector $(\Xi=\Theta)$} \\
\hline I & $\{-1.0,0.0,1.0\}$ & $\{0,0,0,1,1,1\}$ \\
\hline II & $\{-1.0,-0.5,0.5,1.0\}$ & $\{0,0,0,0.5,1,1,1\}$ \\
\hline \multirow{2}{*}{ III } & $\{-1.0,-0.75$, & $\{0,0,0.25,0.5,0.75$, \\
& $-0.25,0.25,0.75,1.0\}$ & $1,1,1\}$. \\
\hline
\end{tabular}

${ }^{\dagger}$ Knot vector is said to be open if the values of its first and last knots appear $(\mathrm{p}+1)$ times. 


\begin{tabular}{c|l|l}
\hline Case & Control points $(\mathrm{C})$ & \multicolumn{1}{|c}{ Knot vector $(\Xi=\Theta)$} \\
\hline \multirow{4}{*}{ IV } & $\{-1.0,-0.875,-0.625$, & $\{0,0,0,0.125,0.25$, \\
& $-0.375,-0.125,0.125$, & $0.375,0.5,0.625$, \\
& $0.375,0.625,0.875$, & $0.75,0.875,1,1,1\}$. \\
& $1.0\}$ & \\
\hline
\end{tabular}

As illustrated in Figure 6, four cases with 1, 4, 16, 64 cells are used to form the stiffness matrix for IGA. From Case I to Case IV, we increase the numbers of knot and control point but we fixed the degree of basis function during the analysis. Therefore, the size of structural stiffness term will be $\mathrm{K}_{9 \times 9}^{\text {case I }}, \mathrm{K}_{16 \times 16}^{\text {case II }}, K_{36 \times 36}^{\text {case III }}, K_{100 \times 100}^{\text {case IV }}$ for four cases respectively.
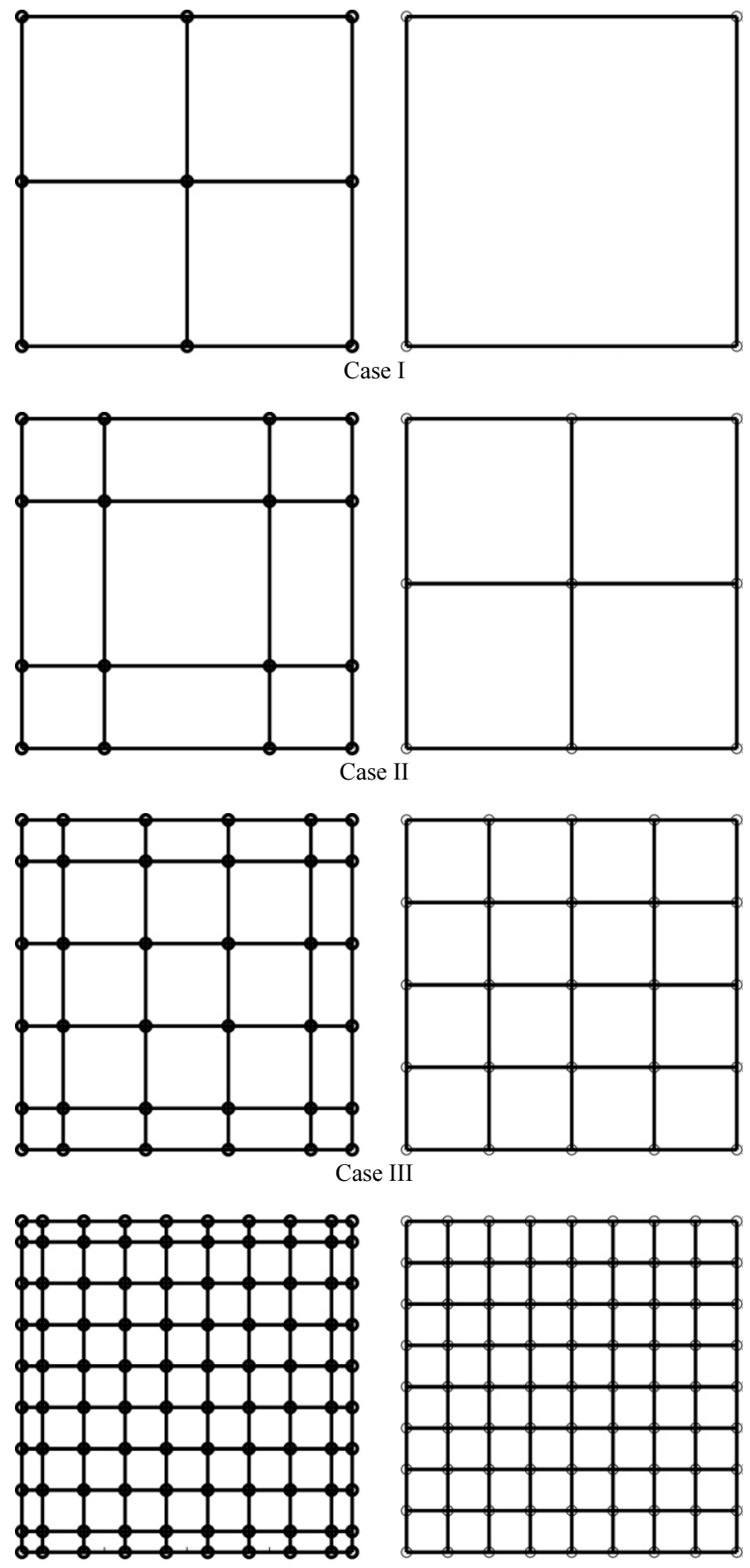

Case IV

Figure 6. Square plate: (left) Control points net and (right) mesh for IGA
From IGA, we obtain temperature distributions for four cases. The maximum temperature is detected at centre of the plate. The temperatures distributions are very similar to each other. Maximum temperature values of $0.3125,0.3125,0.29539$ and 0.29473 are calculated from Case I, II, III and IV. In the last, we compare all the solutions produced in this example. For clear comparison, we are sampling the temperature values along the line with the values $y=0$ and illustrated in Figure 7. From the graph, we can see the IGA produces an acceptable maximum value of temperature with only 1 cell and also with 4 cells. In addition, the IGA with 16 cells provides very accurate temperature values along the line. As described earlier, FE solution uses 256 bilinear FEs and 64 biquadratic FEs to produce similar solution with analytical solution. It means that 1024 loops for numerical integration is required for the $\mathrm{FE}$ analysis with Q1 but IGA with 16 cells needs only 144 loops when $3 \times 3$ Gauss integration rule is used.

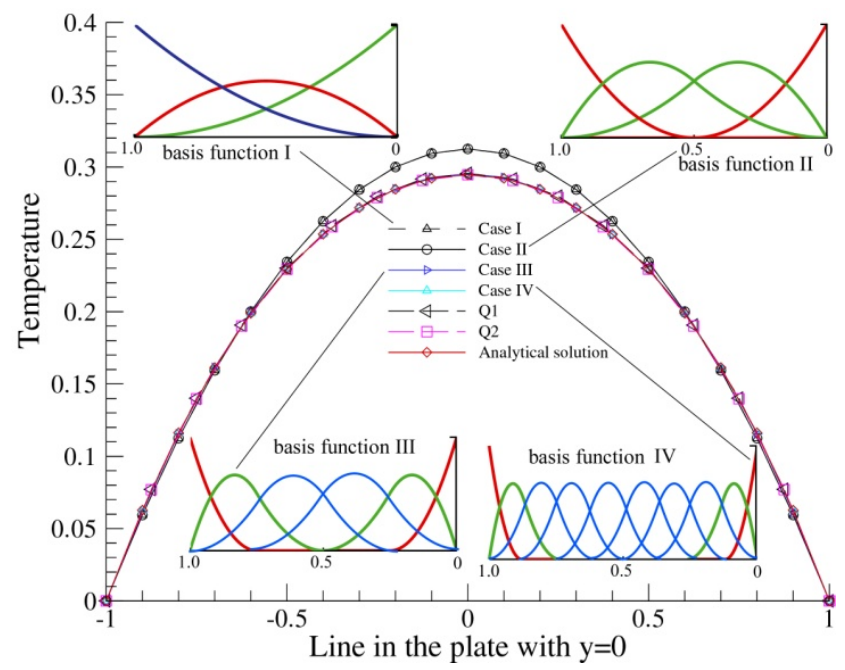

Figure 7. Temperature distribution of plate from IGA

\subsection{L-shape plate}

L-shape plate with constant load function $\mathrm{f}(\mathrm{x})=1$ with Dirichlet boundary condition is here considered. The geometry of L-shape plate is illustrated in Figure 8.

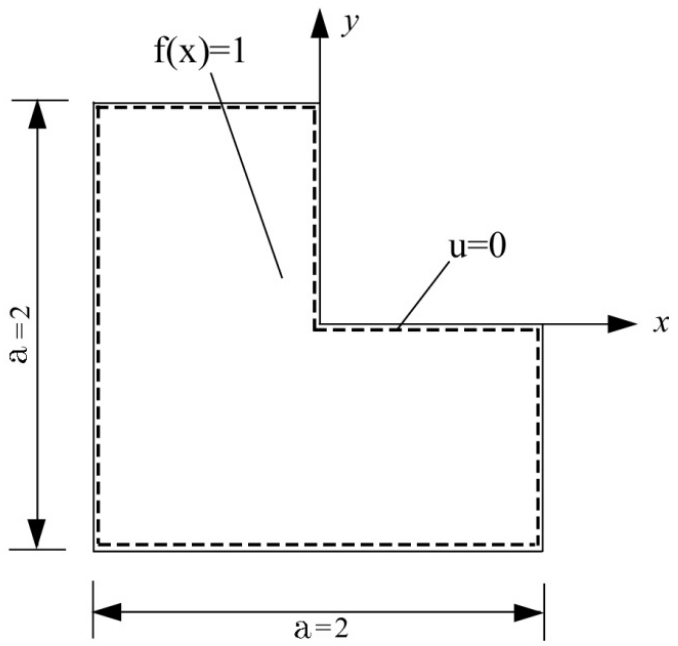

Figure 8. L-shape plate subjected to uniform heat load with Dirichlet boundary condition 
Prior to IGA, FE solutions are also prepared by using bilinear and biquadratic FEs (Q1 and Q2). For Q1 FE, 16 FEs per plate side are used in the analysis and 8 FEs per plate side are used for Q2 FE as shown in Figure 9. Both FEs produces very similar solutions: Q1 and Q2 produce the values of 0.14725 and 0.14755 respectively at two positions $(-0.375,-0.25)$ and $(-0.25,-0.375)$ of plate. The locations of maximum temperature values are marked in Figure 9.

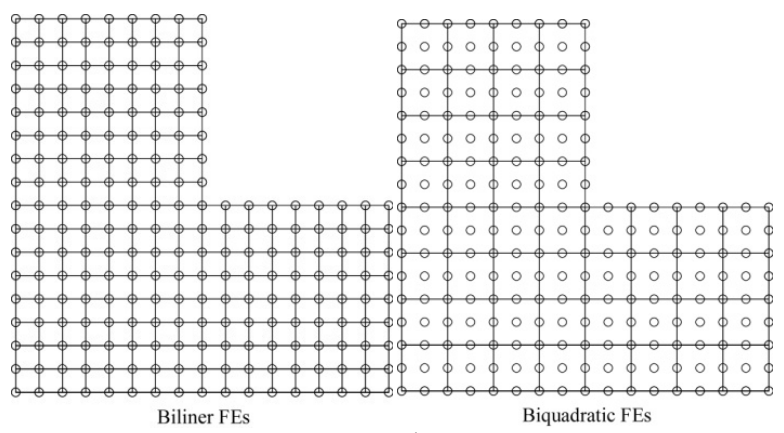

FE meshes

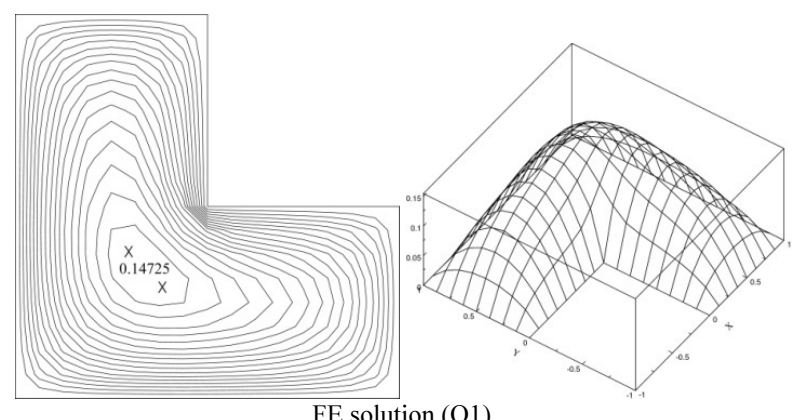

FE solution $(\mathrm{Q} 1)$

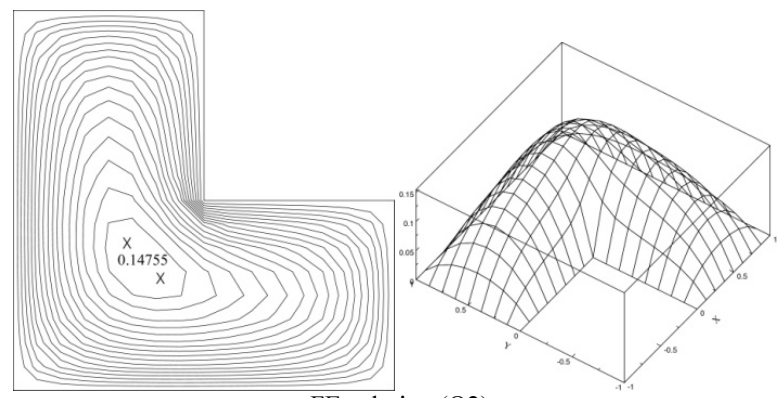

FE solution (Q2)

Figure 9. FE mesh and solutions of L-shape plate subjected to uniform heat load by using bilinear FE (Q1) and biquadratic FE (Q2)

There are various ways to parameterize the L-shape plate using NURBS. In this example, we use two different continuities such as $\mathrm{C}^{0}$ and $\mathrm{C}^{1}$.

First, the knot vectors $\Xi=\{0,0,0,0.5,0.5,1,1,1\}$ and $\Theta=\{0,0,0,1,1,1\}$ are used to achieve $\mathrm{C}^{0}$ continuity of basis function for Case I. Subsequently, other three cases are analyzed with the same $\mathrm{C}^{0}$ continuity. The control points for Case I and knot vectors for Case II, III and IV are summarized in Table 2.
Table 2. Control points or/and Knot vector

\begin{tabular}{c|l}
\hline Case & Control points $(\mathrm{C})$ or/and Knot vector $(\Xi, \Theta)$ \\
\hline \multirow{2}{*}{ I } & $C=\{1-1 ; 0-1 ;-1-1 ;-10 ;-11 ; 1-0.6 ; 0-0.55 ;-0.5-0.5 ;-0.550 ;$ \\
& $-0.651 ; 10 ; 0.50 ; 00 ; 00.5 ; 01\}$ \\
\hline \multirow{2}{*}{ II } & $\Xi=\{0,0,0,0.25,0.5,0.5,0.75,1,1,1\}$, \\
& $\Theta=\{0,0,0,0.5,1,1,1\}$ \\
\hline \multirow{3}{*}{ III } & $\Xi=\{0,0,0,0.25,0.5,0.75,1,1,1\}$, \\
& $\Theta=\{0,0,0,0.125,0.25,0.375,0.5,0.5$ \\
& $0.625,0.750 .875,1,1,1\}$ \\
\hline \multirow{4}{*}{ IV } & $\Xi=\{0,0,0,0.125,0.25,0.375,0.5,0.625$, \\
& $0.75,0.875,1,1,1\}$ \\
& $\Theta=\{0,0,0,0.0625,0.125,0.1875,0.25,0.3125$, \\
& $0.375,0.6875,0.75,0.8125,0.875$, \\
& $, 0.9375,1,1,1\}$
\end{tabular}

For IGA, the meshes with 2, 8, 32, 128 cells as shown in Figure 10 are used for case I, II, III, IV respectively.
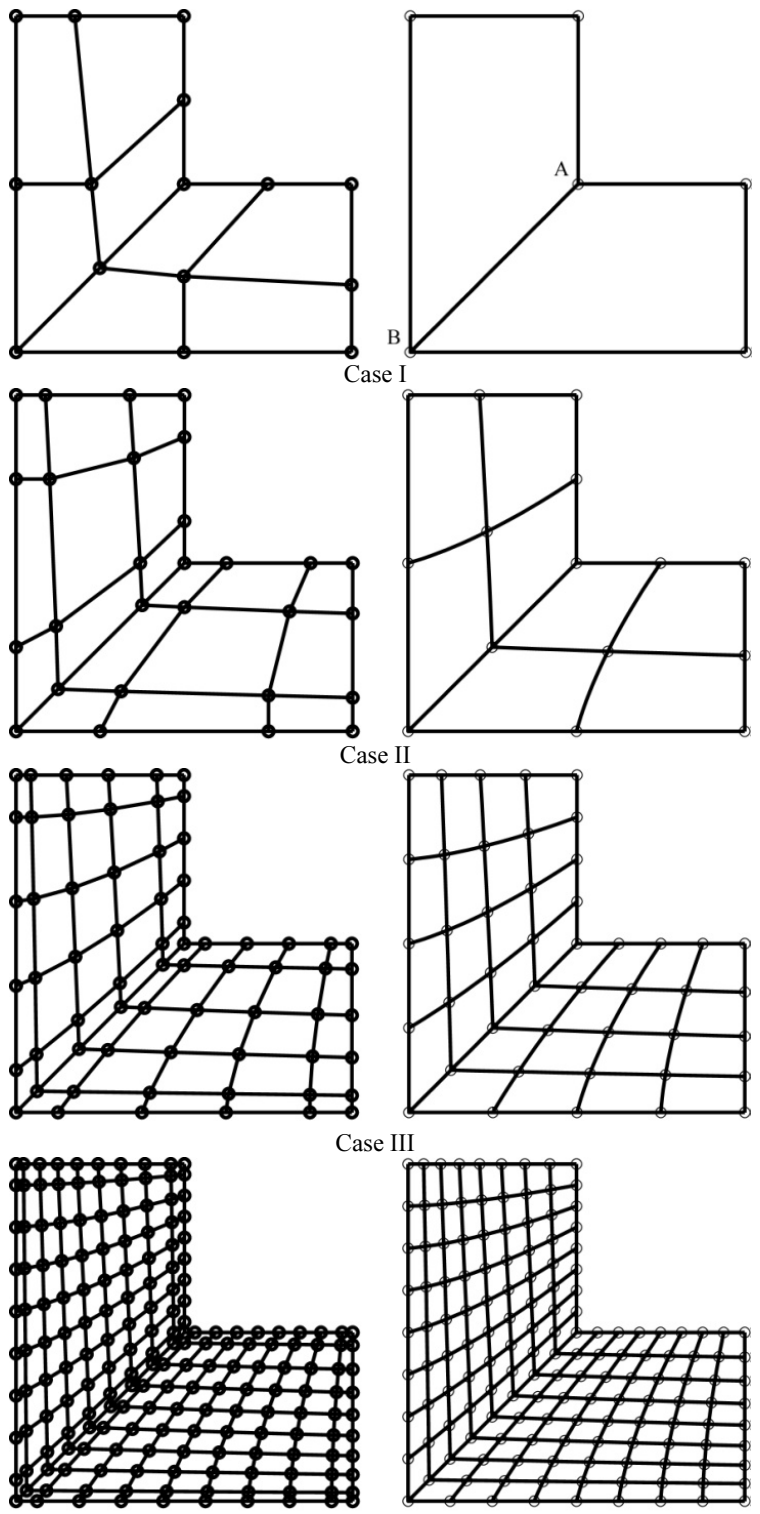

e III

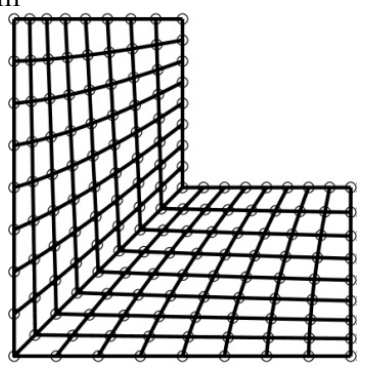

Case IV

Figure 10. L-shape plate: (left) Control point net and (right) mesh for IGA with $\mathrm{C}^{0}$ continuity 
From IGA of L-shape plate, we can see the temperature distribution become smoother all over the plate with larger cell number. However, with small number of cell, the Case I produces a slight discontinuity near the line $\overline{\mathrm{AB}}$ since the $\mathrm{C}^{0}$ continuity is satisfied. However, it is found to be that this discontinuity gradually disappears when the number of cell is increased for analysis. The temperature distributions for all four cases are illustrated in Figure 11. Maximum value of temperature 0.13019 are detected at the positions $(-0.51,-0.24)$ and $(-0.24,-0.51)$ for Case I. For Case II, III and IV, the maximum values of $0.14983,0.14808,0.14851$ are calculated at the position $(-0.35,-0.35)$.
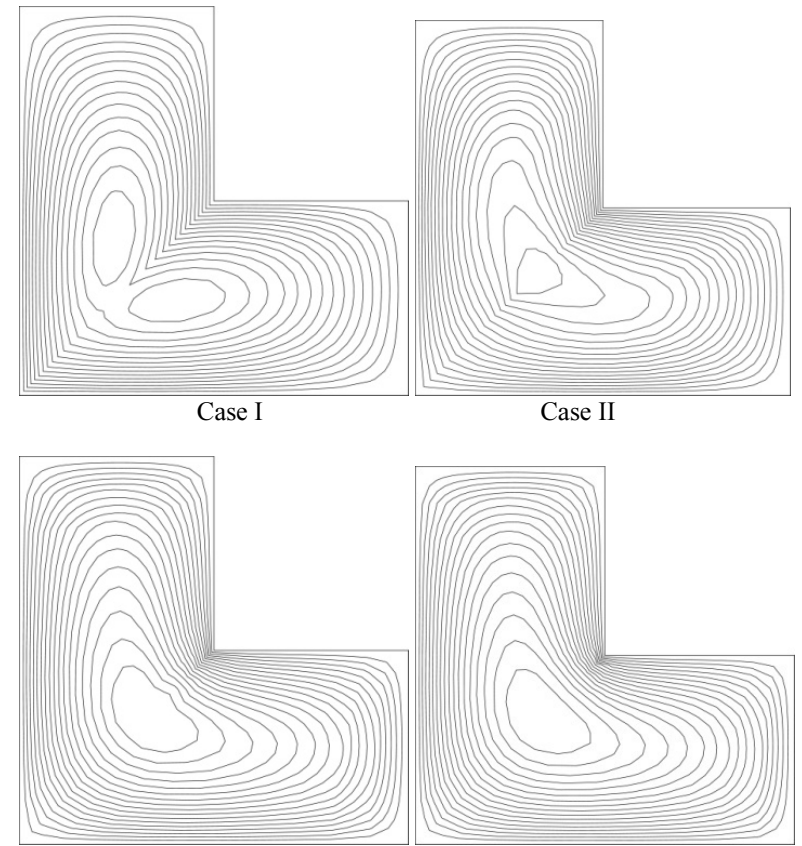

$$
\text { Case III }
$$

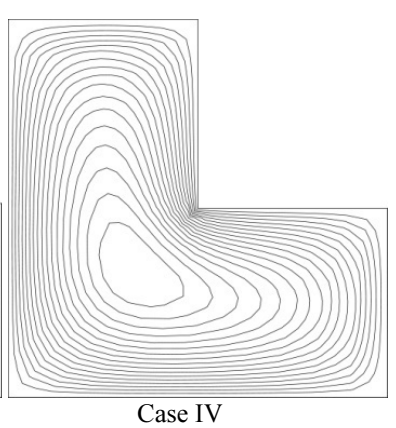

Figure 11. Temperature distributions of L-shape plate from IGA with $\mathrm{C}^{0}$ continuity

Second, the knot vector $\Xi=\{0,0,0,0.5,1,1,1\}$ and $\Theta=\{0,0,0,1,1,1\}$ is used for $\mathrm{C}^{1}$ continuity. Control points for Case $\mathrm{V}$ and knot vectors for other cases such as Cases VI, VII, and VIII are summarized in Table 3. Note that some control points are repeatedly used to form control point net and those are marked in Figure 12.

Table 3. Control points and Knot vector

\begin{tabular}{|c|c|}
\hline Case & Control points $(\mathrm{C})$ or/and Knot vector $(\Xi, \Theta)$ \\
\hline \multirow[t]{2}{*}{$\mathrm{V}$} & $\begin{array}{l}C=\{-1-1 ;-11 ;-11 ; 11 ;-0.6-1 ;-0.70 ; 00.7 ; \\
10.6 ; 0-1 ; 00 ; 00 ; 10\}\end{array}$ \\
\hline & $\Xi=\{0,0,0,0.5,1,1,1\}$ and $\Theta=\{0,0,0,1,1,1\}$ \\
\hline VI & 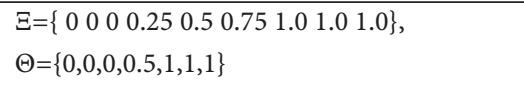 \\
\hline VII & 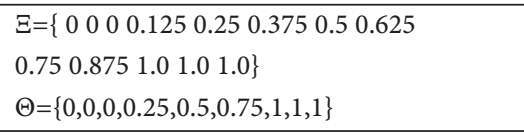 \\
\hline VIII & 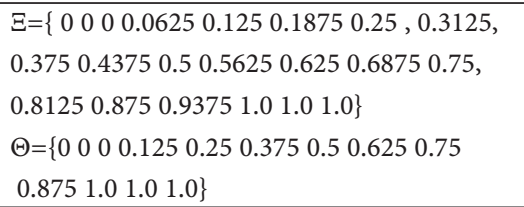 \\
\hline
\end{tabular}
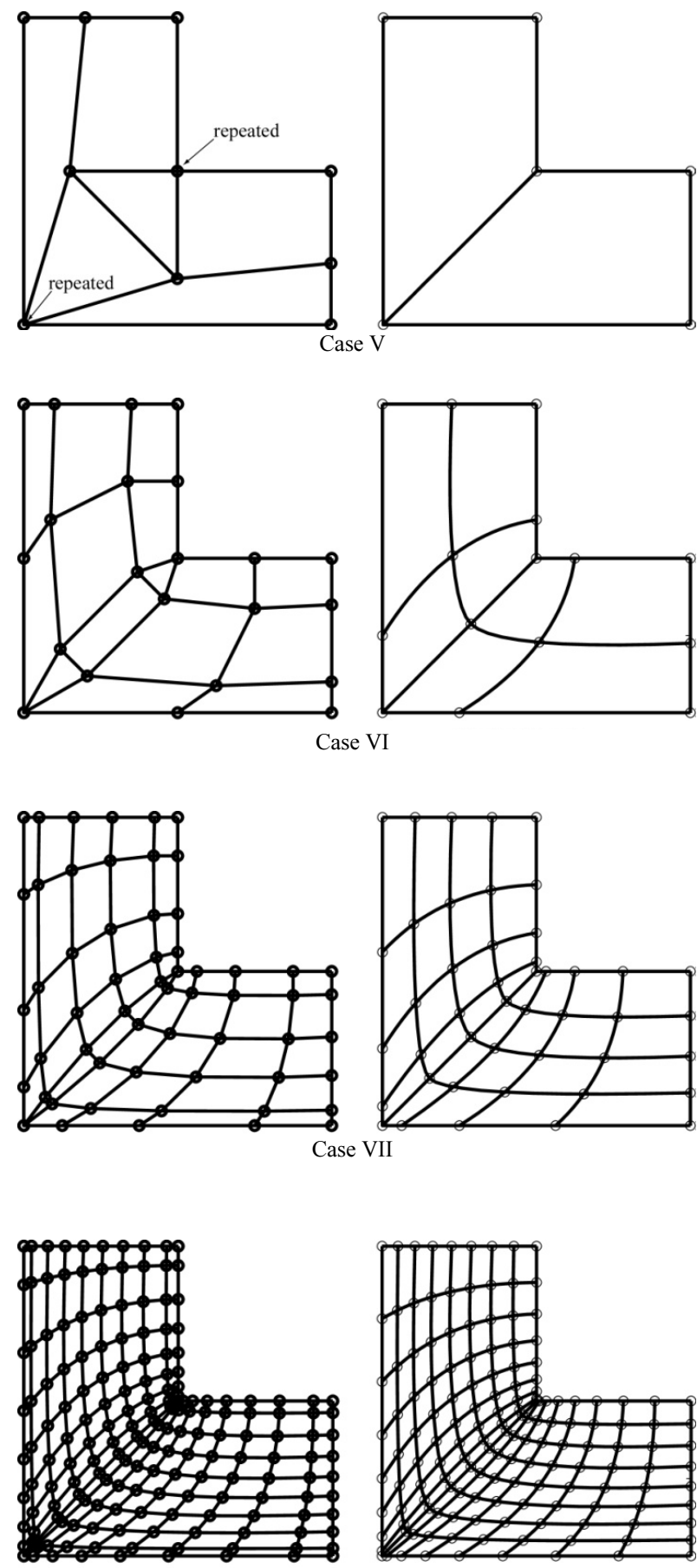

Case VIII

Figure 12. L-shape plate: (left) Control point net and (right) mesh for IGA with $\mathrm{C}^{1}$ continuity

For IGA, the meshes with 2, 8, 32, 128 cells are used for Cases V, VI, VII, and VIII. From comparisons with Cases I IV, the cases with $\mathrm{C}^{1}$ continuity can provide smoother temperature distribution with even coarse mesh. Maximum value of temperature 0.13423 are detected at the positions $(-0.425,-0.425)$ of plate for Case V. For Case VI, VII and VIII, the maximum values of 0.14785 , 0.14798 and 0.14923 are calculated at the same position $(-0.32725$, $-0.32725)$. The temperature distributions for all four cases with $C^{1}$ continuity are illustrated in Figure 13. 

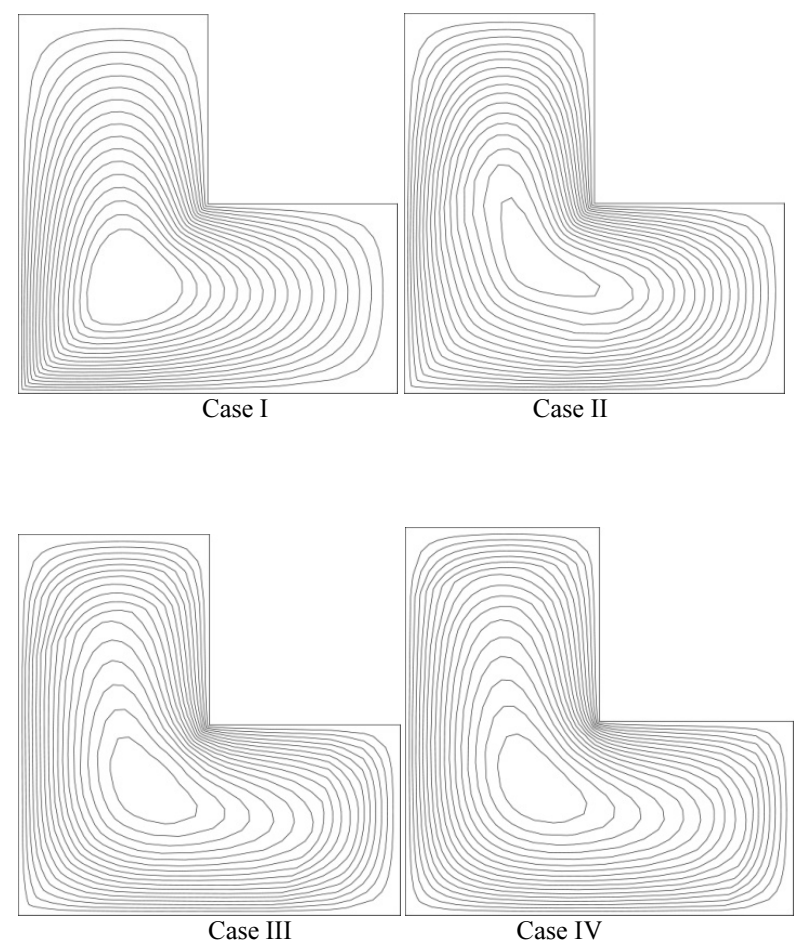

Figure 13. Temperature distributions of L-shape plate from IGA with $\mathrm{C}^{1}$ continuity

\section{CONCULSIONS}

The isogeometric concept is successfully introduced in solving Poisson equation. The procedures used in isogeometric formulation are described in this paper. Two numerical tests are carried out to show the validity of isogeometric formulation we described. Here, some specific conclusions are drawn from numerical tests using IGA:

1. It is found to be that isogeometric concept can guarantee very accurate geometric definition based on B-spline surface.

2. Isogeometric formulation can achieve the complete integration between geometry and analysis.

3. It is very easy to manipulate the structural geometry in IGA and no much effort is required to perform $h$-adaptive analysis.

4. Lower continuity of basis function can produce some discontinuity in solution field with coarse mesh so that an appropriate size of control net seems to be prepared in IGA.

5. It turned out to be that more accurate geometric definition can lead to better temperature distribution especially for irregular shape of structure.

6. Numerical integration rule could be important issue in IGA and further research is therefore required. In particular, the investigation on which types of numerical integration rule could be the best for IGA will be crucial.

Finally, two numerical tests provided in this paper are provided as future reference solutions.

\section{REFERENCES}

de Boor, C. (1978) "A practical guides to splines." New York, Springer-Verlag.

Cottrell, J.A., Bazilevs, Y. and Hughes, T.J.R. (2009) "Isogeometric Analysis: Towards Integration of CAD and FEA." Wiley.

Hughes, T.J.R. (1987) "The finite element method linear static and dynamic finite element analysis." Prentice-Hall, New Jersey

Hughes, T.J.R., Cottrell, J.A. and Bazilevs, Y. (2005) "Isogeometric analysis: CAD, finite elements, NURBS, exact geometry and mesh refinement." Comput. Methods Appl. Mech. Engrg., 194: $4135-4195$

Hughes, T.J.R. and Evans J.A. (2010) "Isogeometric analysis." ICES Report 10-18, The Institute of Computational Engineering and Science, University of Texas Austin

Irons, B. M. and Zienkiewicz, O. C. (1968) "The isoparametric finite element system - a new concept in finite element analysis." Proc. Conf. Recent Advances in Stress Analysis, Royal Aeronautical Society.

Kim, Y. M. (1984) "An elementary structural analysis of finite element method using cubic B-spline., Proc. Annual Conf. of AIK, Architectural Institute of Korea.

Mizusatia, T., Kajita, T. and Naruoka, M. (1979) "Vibration of skew plates by using B-spline functions." J. Sound Vibration, 62(2):301-308

Zamani, N.G. (1981) "A least squares finite element method applied to b-splines." Journal of the Franklin Institute, 311(3): 195-208

(Date of Submission : 2010.7.3) 